\title{
General Derivation of Mass-Energy Relation without Electrodynamics or Einstein's Postulates
}

\author{
Mario Rabinowitz \\ Armor Research, Redwood City, CA, USA \\ Email: mario715@gmail.com
}

Received 4 May 2015; accepted 8 August 2015; published 11 August 2015

Copyright (C) 2015 by author and Scientific Research Publishing Inc.

This work is licensed under the Creative Commons Attribution International License (CC BY). http://creativecommons.org/licenses/by/4.0/

c) (i) Open Access

\section{Abstract}

The mass-energy equation $E \approx m c^{2} \approx \frac{m_{0} c^{2}}{\sqrt{1-v^{2} / c^{2}}}$ is derived in general from Newton's equation of motion without use of electrodynamics, or Einstein's Postulates which were presented in his superb 1905 paper on Special Relativity (SR). This was previously not thought to be possible. This novel derivation of an accelerated body of rest mass $m_{0}$ is compared with the traditional SR inertial derivation. A discussion is given of pre-1905, electrostatic and electrodynamic derivations of the mass-energy relation yielding $E \approx m c^{2}$, as well as more recent ones. A concise pre-relativity history of the mass-energy relation is traced back to Newton in 1717.

\section{Keywords}

Mass-Energy Relation, Mass Variation, Inertia, Einstein, Newton, Special Relativity, Mass-Energy Origin, Mass-Energy History

\section{Introduction}

In 1687 when Newton introduced mass in his $2^{\text {nd }}$ law of motion [1], the word mass was limited to the concept of a body's inclination to resist changes in its state of motion. This tendency is known as inertia. Since then the concept of mass has evolved to include both such resistance, and the concept of the inter-convertibility of mass and energy. In the latter abstraction, the mass of a body is a measure of its energy; and equivalently the energy of a body is a measure of its inertial mass. This is evidenced by the famous relation $E=m c^{2}$, where $E$ is the energy equivalent of the mass $m$, and $c$ is the velocity of light in free space. It is generally thought that such a 
relationship between mass and energy is strictly a consequence of relativistic physics. As will be shown in this paper, this belief is not well founded.

\section{Derivation of Mass-Energy Relation}

\subsection{A Concise History of the Sources of $E \approx m c^{2}$}

There have been many electrostatic and electromagnetic derivations of the mass-energy relation. The electrodynamic derivations primarily relied on the work of J. H. Poynting [2]. In 1884 he used Maxwell's equations, to derive the time rate of energy transfer for an electromagnetic field per unit area as

$$
\boldsymbol{S}=\boldsymbol{E} \times \boldsymbol{H},
$$

where $\boldsymbol{S}$ is the Poynting vector, $\boldsymbol{E}$ is the electric field vector, and $\boldsymbol{H}$ is the magnetic field vector. This is the commonly used Abraham form of the Poynting vector in terms of $E \& H$ rather than the Minkowski form in terms of $D \& B$. Although this makes no difference in free space, it makes a difference in electromagnetic energy flux transfer in a solid.

One historical approach uses the Poynting vector to yield the electromagnetic momentum of a point-like charge moving with velocity $v$ :

$$
\boldsymbol{p}=\frac{1}{c} \int \boldsymbol{E} \times \boldsymbol{H} \mathrm{d} V
$$

where $V$ is the volume occupied by the electromagnetic field. If the point-like charge (such as an electron) is moving in the x-direction, by symmetry $p_{y}=p_{z}=0$ and

$$
p_{x}=\frac{1}{c} \int\left(E_{y} H_{z}-E_{z} H_{y}\right) \mathrm{d} V .
$$

Combined with Newton's mechanics, these approaches led to the mass-energy relation $E=m c^{2}$ with velocity dependent masses.

More than a century ago prominent physicists combined Maxwell's equations with Newton's equation of motion to obtain velocity dependent masses before Einstein's awesome 1905 paper [3]. Starting in 1881, J. J. Thomson pointed out that the inertia of a charged body is greater than a neutral body because of the additional inertia of its electromagnetic field. This concept was refined by O. Heaviside in 1889; and later by G. F. C. Searle in 1897 following Henrik Lorentz' extensively detailed paper in 1892 [4] in which he had many more than 185 equations (only 185 were numbered). Thomson, Searle and O. Heaviside (1889) separately derived an increase in the electromagnetic inertial mass of a charged body as its velocity increases. In 1903, Max Abraham [5] was one of the first [although he is considered the first, he references Lorentz (1900) on $m_{L}$ and $m_{T}$ ] to introduce $m_{L}$ and $m_{T}$ that became codified as:

$$
\begin{aligned}
& \text { Longitudinal mass } m_{L}=\frac{m}{\left(\sqrt{1-v^{2} / c^{2}}\right)^{3}} \text {, and } \\
& \text { Transverse mass } m_{T}=\frac{m}{\sqrt{1-v^{2} / c^{2}}} \text { (where } m \text { [sic] is the rest mass). }
\end{aligned}
$$

In 1889 George Fitz Gerald explained the null result of the 1887 Michelson-Morley experiment by introducing a length contraction factor $\sqrt{1-v^{2} / c^{2}}$. A year before in 1888, Oliver Heaviside (using Maxwell's equations, which he earlier recast from the original cumbersome 20 equations with 20 unknown variables, into 4 differential vector equations with 2 variables) compared the electric and magnetic fields of a point-like charge and an equally charged ellipsoid. Remarkably, Heaviside found that the fields of a steadily moving (mathematically constructed) contracted ellipsoid having axes in the ratios $\sqrt{1-v^{2} / c^{2}}: 1: 1$ are the same as those of a similarly moving point charge, $v$ being the velocity of both the ellipsoid and the point charge in free space.

Lorentz found that experiments on fast moving electrons gave results that were not consistent with Abraham's model of a rigid charged spherical conductor. In 1904, Lorentz, assuming an ether throughout absolute space, found similar results to FitzGerald from what is known as the Lorentz Transformation which is integral to Eins- 
tein’s 1905 [3] introduction of Special Relativity. In 1905 H. Poincare proved that non-electromagnetic forces are needed to keep the electrostatic forces from causing an electron to fly apart. Abraham and Lorentz based their calculations on particular electromagnetic models of the electron.

Einstein used the concepts of longitudinal and transverse mass in his 1905 paper [3], but derived an inappropriate form of transverse mass

$$
m_{T}=\frac{m}{1-v^{2} / c^{2}}
$$

The electrodynamic masses of Equations (3) and (4), as well as Einstein's 1905 longitudinal and transverse masses all have been abandoned, although some would argue that Equations (3) and (4) are valid relativistic mass concepts. Subsequent to his 1905 paper, Einstein showed by means of his postulates that the velocity dependence of mass does not depend on electromagnetics or structural details of a charged body such as an electron, but is totally general. He also eliminated the ether and absolute space, but may have inadvertently reintroduced the ether in his theory of General Relativity by imbuing space with properties such as curvature and metric expansion.

So, there is now only the Einstein "relativistic mass" which is the same form as $m_{T}$ of Equation (4). Some scholars think that relativistic mass should also be abandoned arguing that the increase of energy with velocity originates not in the object but in the geometric properties of space-time itself. Much later Einstein abandoned velocity dependent mass concepts altogether, saying in 1948 that it is not good to introduce the concept of the [relativistic] mass of a moving body for which no clear definition can be given.

From the past to the present, the Energy-Mass relation $E \approx m c^{2}$ was obtained by many differing electrostatic and electromagnetic considerations - and related to the mass of a stationary or moving point-like charge. Dating back more than a century this was done by H. Lorentz 1892, J. J. Thomson 1897, M. Abraham 1903, H. Poincaré 1905, and other distinguished physicists. In 1904, Fritz Hasenöhrl showed that electromagnetic radiation enclosed in a cavity contributes to the inertia $m \approx E / c^{2}$ of the cavity [6]. In at least two more papers [7] [8], Hasenöhrl sharpened his analysis to include stress in the cavity. But he did not generalize to mass in general. Later in 1905 (and since 1905) Einstein derived $m=E / c^{2}$, as well as other physicists with additional complexities. Some have even constructed higher dimensional electromagnetic mass models; but higher dimensions are not observable [9]. In 2012 S. Hajra [10] starting from the electromagnetic momentum of Helmholtz, Abraham, and Lorentz got $m=\frac{m_{0}}{\sqrt{1-v^{2} / c^{2}}}$ and $E=m c^{2}$. Except for Hasenöhrl's radiation mass, the above derivations are restricted to the mass of the electromagnetic or electrostatic field of a point-like charge. The validity of all these depends on the exclusion of neutral bodies.

Despite Einstein's 1948 comments, many distinguished relativity experts such as M. Born, V. Fock, F. Klein, M. Laue, and R. Tolman in the past; and W. Rindler, and R. Penrose in relatively recent times use the concept of relativistic mass, and it is still taught. The intent of the present paper is not to take sides on the dispute of whether one should or should not use the concept of relativistic mass. Both sides will agree that in Section 2.3 the basic undisputed finding of Equation (14) $\sum_{\alpha=0}^{3} P_{\alpha}^{2} \equiv P^{\alpha} P_{\alpha}=m^{2} c^{2}=m^{2} v^{2}+m_{0}^{2} c^{2}$ means that the energymomentum four-vector has a fixed magnitude $m c$. Yet as shown in Section 2.3, the disputed relativistic mass $m=\frac{m_{0}}{\sqrt{1-v^{2} / c^{2}}}$ is related to Equation (14) by simple algebra. One object of this paper is to show that this result can be derived simply from Newton's equation of motion. The significance of this finding means this result is more general than previously thought; and may even have relevance to accelerated systems and quantum mechanics where the concept of mass at times appears nebulous as it does to some degree in zitterbewegung.

\subsection{Simple Newtonian Approach}

Choosing the Feynman route (approach) to investigate the available options for the mass-energy relation, we begin with the classical Newtonian equation of motion for single, isolated particles. Although deserving of attention, to the author's knowledge this simple option has not yet been treated in the literature. The main goal of this derivation is to show that the mass-energy relation can simply be derived from Newton's equation. 
Let us derive the mass-energy relation $m$ of a body of rest mass $m_{0}$, as it accelerates from velocity $v_{0}=0$ in its rest (proper) frame to velocity $v$ relative to an observer by a force $F$. From electromagnetic considerations as discussed above [or for Newton's light corpuscles with the dimensionless number $f=\frac{1}{2}$, cf. Section 3] we can assume in general that

$$
E=f m c^{2}
$$

is the energy of the body that can increase with acceleration. The constant $c$ is the speed of light now known to be invariant in all frames. If this derivation were done long before Einstein's 1905 SR paper [3], for a Galilean frame $(c \pm v)^{2} \approx c^{2}$ for $c \gg v$ and Equation (6) can take this into account with the number $f$. After obtaining $m$, we can determine if the result for $f m c^{2}$ is compatible with known physics. If so, the assumption is both self-consistent and isomorphic with experimental observations, and independent of Einstein's postulates of Special Relativity requiring inertial frames, and regarding the velocity of light. That $c^{2}$ is an excellent approximation as input to Equation (7), even at very high velocities, could be warranted by electron experiments prior to 1905.

The derivation here uses Newton's equation of motion cast in the form of a power equation. Thus it differs from Einstein's approach [3], and also with the inclusion of the $\mathrm{d} m / \mathrm{d} t$ term.

$$
\begin{aligned}
& \frac{\mathrm{d} E}{\mathrm{~d} t}=F \cdot v \rightarrow \frac{\mathrm{d}\left(f m c^{2}\right)}{\mathrm{d} t}=\left[m \frac{\mathrm{d} v}{\mathrm{~d} t}+v \frac{\mathrm{d} m}{\mathrm{~d} t}\right] v \rightarrow f c^{2} \frac{\mathrm{d} m}{\mathrm{~d} t}=m v \frac{\mathrm{d} v}{\mathrm{~d} t}+v^{2} \frac{\mathrm{d} m}{\mathrm{~d} t} \\
& \rightarrow \int_{m_{0}}^{m} \frac{\mathrm{d} m}{m}=\int_{v_{0}}^{v} \frac{v \mathrm{~d} v}{\left(f c^{2}-v^{2}\right)} \rightarrow \ln \left(\frac{m}{m_{0}}\right)=\frac{-1}{2}\left\{\ln \left[1-\frac{v^{2}}{f c^{2}}\right]\right\} .
\end{aligned}
$$

With $v_{0}=0 \Rightarrow m_{0}$ is the rest mass in the rest (proper) frame. So Equation (7) $\Rightarrow$

$$
m_{a c c} \equiv m=\frac{m_{0}}{\sqrt{1-v^{2} / f c^{2}}} .
$$

This author first derived $m_{a c c} \equiv m \approx \frac{m_{0}}{\sqrt{1-v^{2} / f c^{2}}}$ for $v \ll c$, without $\frac{\mathrm{d} m}{\mathrm{~d} t}$ and then realized that the $\mathrm{d} m / \mathrm{d} t$ term gives an exact result. In either derivation, the velocity $v$ can be time varying, since there was no restriction that the velocity $v$ is constant as for an inertial frame.

Using Equation (8) for $m_{a c c}$, assuming $E=f m c^{2}, f m_{a c c} c^{2}$ leads to a result that agrees with known physics and this can easily be demonstrated. In advance there was no guarantee that this would be the case. Multiplying Equation (8) by $c^{2}$ :

$$
E=f m c^{2}=f m_{0} c^{2}\left[1-\frac{v^{2}}{f c^{2}}\right]^{-1 / 2}
$$

with $\frac{1}{2} \leq f \leq \frac{4}{3}$ from Newton's corpuscles to electrodynamics; and confirmed at high velocities for $f=1$. For $v \ll c$, a binomial expansion of Equation (9) yields the well-known result:

$$
E=f m_{0} c^{2}\left[1+\frac{1}{2} \frac{v^{2}}{f c^{2}}+\frac{3}{8} \frac{v^{4}}{f^{2} c^{4}}+\cdots\right] \approx f m_{0} c^{2}+\frac{1}{2} m_{0} v^{2}=m_{0} c^{2}+\frac{1}{2} m_{0} v^{2}
$$

Therefore the assumption $E=m c^{2}$ with $f=1$ can be justified as a starting point independent of Special Relativity. Note that the low velocity equation for kinetic energy, $K E=\frac{1}{2} m_{0} v^{2}$ is unchanged regardless of the value of $f$. If an incorrect mass such as either the longitudinal mass $m_{L}=\frac{m}{\left(\sqrt{1-v^{2} / c^{2}}\right)^{3}}$ of Equation (3) or 
the transverse mass of Equation (5) obtained by Einstein [3] in 1905 had been used to test the energy-mass equation $E=m c^{2}$ for self-consistency, and isomorphism with physics data it would have failed. For $v \ll c$, the magnitude of $f$ can be experimentally tested in the quartic term.

\subsection{Traditional Special Relativity (SR) Approach}

We will briefly sketch the traditional SR approach to point out the differences between it and the derivation given above using Newton's equation.

In a space-time of 3 spatial dimensions and 1 dimension of time, the 4-momentum is

$$
P_{\alpha}=m V_{\alpha}
$$

where the 4-velocity

$$
V_{\alpha}=\left[\frac{c}{\sqrt{1-v^{2} / c^{2}}}, \frac{v}{\sqrt{1-v^{2} / c^{2}}}\right] .
$$

The dot product of the vector velocity is the scalar $v^{2}$ in the denominator of (12): $\boldsymbol{v} \cdot \boldsymbol{v}=v^{2}$.

Using the Einstein summation notation

$$
\sum_{\alpha=0}^{3} V_{\alpha}^{2} \equiv V^{\alpha} V_{\alpha}=c^{2} .\left[-c^{2} \text { in some conventions. }\right]
$$

Combining Equations (11) and (13), we have the well-known equation:

$$
\sum_{\alpha=0}^{3} P_{\alpha}^{2} \equiv P^{\alpha} P_{\alpha}=m^{2} c^{2}=m^{2} v^{2}+m_{0}^{2} c^{2}
$$

Dividing Equation (14) by $c^{2}$, and solving for the mass $m$ :

$$
m=\frac{m_{0}}{\sqrt{1-v^{2} / c^{2}}} .
$$

Equation (15) gives the familiar increase of mass with velocity. Although this standard Special Relativity approach is strictly just for inertial frames, it is used for accelerating frames such as with cyclotrons.

\section{Discussion}

In the 1905 paper [3] in which Einstein introduced Special Relativity, he presented his two postulates thus:

"They suggest rather that, as has already been shown to the first order of small quantities, the same laws of electrodynamics and optics will be valid for all frames of reference for which the equations of mechanics hold good. We will raise this conjecture (the purport of which will hereafter be called the 'Principle of Relativity') to the status of a postulate, and also introduce another postulate, which is only apparently irreconcilable with the former, namely, that light is always propagated in empty space with a definite velocity $c$ which is independent of the state of motion of the emitting body."

From context, the first postulate has been interpreted as "inertial frames". The present paper derived the massenergy relation in general terms without recourse to Einstein's postulates.

Reference [11], derived an electrodynamic accelerating mass $m_{a c c}$, and showed its increase with acceleration. The derived equations were fully consistent with $E=m_{a c c} c^{2}$. In addition to demonstrating its relevance to charged particles, a heuristic argument was made that it could also apply to neutral particles like atoms. It has come to my attention that electrodynamic derivations such as in [12] [13] which can be interpreted as $m_{\text {acc }}$ have also been made. Reference [11] differs considerably from their content.

Newton had for his light corpuscles (photons): $E=\frac{1}{2} m v^{2}=f m c^{2}$. [As shown in Section 2.2, the value of $f$ in $f m c^{2}$ doesn’t affect $K E=\frac{1}{2} m v^{2}$ for low velocity.] In 1717, Newton presciently said, “Are not the gross bo- 
dies and light convertible into one another...?” [14] In the late 1600’s Isaac Newton and Gottfried Leibniz independently discovered Calculus, and Newton's 1687 Principia [1] was filled with calculus. In 1676 Ole Roemer ingeniously measured the speed of light $c \sim 2.2 \times 10^{8} \mathrm{~m} / \mathrm{sec} \quad\left[0.73\right.$ of the correct value $3 \times 10^{8} \mathrm{~m} / \mathrm{sec}$ ] by timing eclipses of Jupiter's moon Io by Jupiter when the Earth was closest to Jupiter and 6 months later (on the opposite side of the Sun from Jupiter) thus increasing light's traversal distance by the Earth's orbital diameter. It was thus known that $c \gg$ any other known speed. Thus it appears that Newton had all he needed to derive the energy-mass relationship $E=f m c^{2}=\frac{f m_{0} c^{2}}{\sqrt{1-v^{2} / c^{2}}} \approx f m_{0} c^{2}+\frac{1}{2} m_{0} v^{2}$ with $f=1 / 2$ by the time of his Opticks in 1717, as demonstrated in Section 2.2. However, he did not do so.

\section{Conclusions}

In simplest terms the derivation of this paper comes from the conservation of total power, which is a consequence of the detailed balance of the conservation of mass-energy. For those who prefer to restrict the concept of mass to rest mass $m_{0}$, then the derivation of Section 2.2 can just as well be considered a derivation of the dependence of energy increase of a body of rest mass $m_{0}$ with acceleration.

This paper derived the generalized mass-energy relationship from Newton's equation of motion cast in the form of a power equation. The result is fully consistent with special relativity. Einstein's postulates were not used regarding the velocity of light, nor that the laws of physics are the same in all inertial frames. If an incorrect mass such as either the longitudinal mass of Equation (3) or the transverse mass of Equation (5) obtained by Einstein [3] in 1905 [and later corrected] had been derived, it would have failed the tests of self-consistency and compatibility with physical data.

It is the great genius of Albert Einstein who accomplished the monumental task of constructing a complete mechanics within the framework of relative space and relative time, and described both mechanical and electrodynamic phenomena in a single stage within that structure.

\section{Acknowledgements}

I wish to acknowledge the countless generous, hard-working, and tireless beings from whom I have learned and gleaned knowledge both directly and indirectly; and upon whose unselfish shoulders I have stood.

\section{References}

[1] Newton, I. (1687) Philosophiæ Naturalis Principia Mathematica [Mathematical Principles of Natural Philosophy] Vol. 1. The Motion of Bodies. Motte, A., Trans., University of California Press, Berkeley.

[2] Poynting, J.H. (1884) Philosophical Transactions of the Royal Society of London, 175, 343-361. http://dx.doi.org/10.1098/rstl.1884.0016

[3] Einstein, A. (1905) Annalen der Physik, 17, 891-921. http://dx.doi.org/10.1002/andp.19053221004

[4] Lorentz, H.A. (1892) Archives néerlandaises des sciences exactes et naturelles, 25, 363-552.

[5] Abraham, M. (1903) Annalen der Physik, 315, 105-179. http://dx.doi.org/10.1002/andp.19023150105

[6] Hasenöhrl, F. (1904) Wiener Sitzungsberichte, 113, 1039-1055.

[7] Hasenöhrl, F. (1904) Annalender Physik, 320, 344-370. http://dx.doi.org/10.1002/andp.19043201206

[8] Hasenöhrl, F. (1905) Annalen der Physik, 321, 589-592. http://dx.doi.org/10.1002/andp.19053210312

[9] Rabinowitz, M. (2014) Advanced Studies in Theoretical Physics, 8, 689-700. http://arxiv.org/abs/1502.04989 http://dx.doi.org/10.12988/astp.2014.4675

[10] Hajra, S. (2012) Journal of Modern Physics, 3, 187-199. http://dx.doi.org/10.4236/jmp.2012.32026

[11] Rabinowitz, M. (2014) Advanced Studies in Theoretical Physics, 8, 1165-1176. http://dx.doi.org/10.12988/astp.2014.411142

[12] Page, L. (1947) Introduction to Theoretical Physics. D. Van Nostrand, New York.

[13] Panofsky, W.K.H. and Phillips, M. (1955) Classical Electricity and Magnetism. Addison-Wesley Publishing Co., Reading, Mass.

[14] Newton, I. (1717) Opticks, or, a Treatise of the Reflections, Refractions, Inflections and Colours of Light, Query 30. 